Article

\title{
Electromagnetic Field Analysis of an Electric Dipole Antenna Based on a Surface Integral Equation in Multilayered Dissipative Media
}

\author{
Yidong Xu ${ }^{1}$, Wei Xue ${ }^{1, *}$, Yingsong $\mathrm{Li}^{1,2, *}$, Lili Guo ${ }^{1}$ and Wenjing Shang ${ }^{1}$ \\ 1 College of Information and Communication Engineering, Harbin Engineering University, Harbin 150001, \\ China; xuyidong@hrbeu.edu.cn (Y.X.); guolili@hrbeu.edu.cn (L.G.); shangwenjing@hrbeu.edu.cn (W.S.) \\ 2 National Space Science Center, Chinese Academy of Sciences, Beijing 100190, China \\ * Correspondence: xuewei@hrbeu.edu.cn (W.X.); Tel.: +86-451-8251-9810; liyingsong@ieee.org (Y.L.)
}

Received: 27 June 2017; Accepted: 27 July 2017; Published: date

\begin{abstract}
In this paper, a novel method based on the Poggio-Miller-Chang-Harrington-Wu-Tsai (PMCHWT) integral equation is presented to study the electromagnetic fields excited by vertical or horizontal electric dipoles in the presence of a layered region which consists of $K$-layered dissipative media and the air above. To transform the continuous integral equation into a block tridiagonal matrix with the feature of convenient solution, the Rao-Wilton-Glisson (RWG) functions are introduced as expansion and testing functions. The electromagnetic fields excited by an electric dipole are calculated and compared with the available results, where the electric dipole antenna is buried in the non-planar air-sea-seabed, air-rock-earth-mine, and multilayered sphere structures. The analysis and computations demonstrate that the method exhibits high accuracy and solving performance in the near field propagation region.
\end{abstract}

Keywords: electromagnetic propagation; layered dissipative media; surface integral equation; PMCHWT

\section{Introduction}

The electromagnetic fields excited by a dipole source in a layered dissipative medium structure have been investigated widely because of their applications in Through-the-Earth communication, underwater communication, ground penetrating radar technology, and antenna design [1-6]. The developments in electromagnetic field propagation in layered medium structures have been analyzed by many investigators, including Wait [7-9] and King $[7,10,11]$ who proposed the asymptotic methods, and the surface-impedance technique has been used for a two-layered region. Contour integrations and ranch cuts have been also used for the electromagnetic field in a layered region $[8,9]$. King et al. have obtained the complete formulas for electromagnetic fields excited by horizontal and vertical electric dipoles in two- and three-layered regions by using the proposed method in the recent studies. However, it is difficult to solve the arbitrary layered region problem. To overcome this drawback, Michalski et al. [12] presented a compact formulation of the electric-type and magnetic-type dyadic Green's functions for a plane-stratified, laterally unbounded multilayered structure, which is based on the tranmission-line network. Nikita et al. [13] has presented a near-fielding radiating dipole antenna next to a three-layered lossy sphere close to a human head model. Moreover, the numerical results have been verified via a unified method of moments $(\mathrm{MoM})$ model based on the electric field integral equation (EFIE) given by Khamas [14]. These algorithms are suitable for analyzing the non-planar electromagnetic problem only when the structure is a concentric sphere, which limits their application in an arbitrary shaped non-planar layered region. Quintana et al. [15] studied the electromagnetic 
field propagation characteristic of shallow water in detail, and the influence of seabed layer is also considered.

In most studies [16-19], surface integral equation (SIE)-based methods have been adopted in the numerical analysis of the scattering of arbitrary shaped objects and the scattering of objects buried in layered structure, but few of them focus on the electromagnetic fields excited by dipoles in arbitrary layered dissipative media. In this paper, an SIE-based method to study the electromagnetic fields excited by electric dipole submerged in arbitrary layered homogeneous dissipative media is proposed. The total electromagnetic fields in each layer with three source components including the excitation source itself, equivalent electric/magnetic surface currents at the top of the layer, and equivalent electric/magnetic surface currents at the bottom of the layer are derived in detail. Then, the formulation proposed for the interface of each layer is discretized by using the Galerkin's method. After that, a matrix equation for a layered dissipative structure with a block tridiagonal matrix feature of a convenient solution is derived [20]. Three application scenarios are constructed and computed to analyze the electromagnetic characteristics with the vertical and horizontal dipole buried in wide, practically important media including sea, wet and other dissipative materials. Finally, the numerical results are presented to discuss the difference of the proposed method in comparison with the Computer Simulation Technology (CST) Studio Suite.

\section{SIEs for Multilayered Dissipative Medium Structures}

A non-planar K-layered dissipative medium structure is illustrated in Figure 1. Let the frequency of excitation sources in homogeneous medium be $\omega$. The electromagnetic constants at the $i$ th-layer are denoted by conductivity $\sigma_{i}$, permittivity $\varepsilon_{i}$, and permeability $\mu_{i}$, with complex wave number $k_{i}$ and wave resistance $\eta_{i}$. Let $S_{i-1}$ and $S_{i}$ denote the top and bottom surfaces of $i$ th-layer medium of the region $\mathrm{D}_{i}$, respectively, where $i=0,1,2, \ldots, K$. In addition, there is no top surface on the half-space region $\mathrm{D}_{0}$ and no bottom surface beneath the half-space region $\mathrm{D}_{K}$.

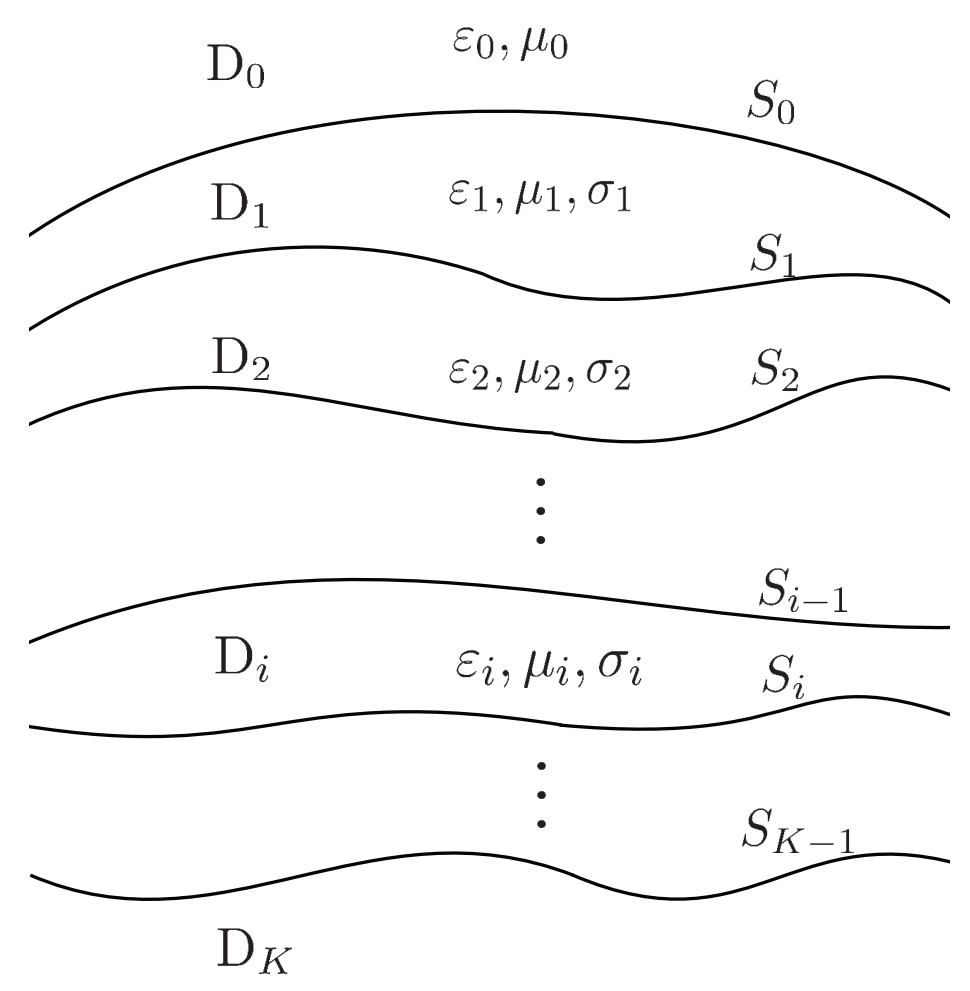

Figure 1. K-layered dissipative medium structure. 
Let $\left(\boldsymbol{E}_{i}^{\text {source }}, \boldsymbol{H}_{i}^{\text {source }}\right)$ denote the incident fields in region $\mathrm{D}_{i}$, which can be excited by electric dipole antennas or magnetic dipole antennas. According to the equivalent principle, the total fields $\left(\boldsymbol{E}_{i}, \boldsymbol{H}_{i}\right)$ in region $\mathrm{D}_{i}$ can be seen as a synergistic result of the incident fields $\left(\boldsymbol{E}_{i}^{\text {source }}, \boldsymbol{H}_{i}^{\text {source }}\right)$, fields $\left(\boldsymbol{E}_{i, i-1}^{\mathrm{JM}}, \boldsymbol{H}_{i, i-1}^{\mathrm{M}}\right)$ excited by the surface currents on $S_{i-1}$, and fields $\left(\boldsymbol{E}_{i, i}^{\mathrm{JM}}, \boldsymbol{H}_{i, i}^{\mathrm{MM}}\right)$ excited by the surface currents on $S_{i}$.

Hence, the total electric and magnetic fields at arbitrary point on surface $S_{i-1}$ or $S_{i}$ can be expressed as

$$
\begin{gathered}
\boldsymbol{E}_{i}=\boldsymbol{E}_{i}^{\mathrm{source}}+\boldsymbol{E}_{i, i-1}^{\mathrm{JM}}+\boldsymbol{E}_{i, i}^{\mathrm{JM}}, \\
\boldsymbol{H}_{i}=\boldsymbol{H}_{i}^{\mathrm{source}}+\boldsymbol{H}_{i, i-1}^{\mathrm{g}}+\boldsymbol{H}_{i, i}^{\mathrm{g}} .
\end{gathered}
$$

The electric and magnetic surface currents on surface $S_{i}$ in region $\mathrm{D}_{i}$ are defined as $\boldsymbol{J}_{i}=\boldsymbol{n}_{i} \times \boldsymbol{H}_{i}$ and $\boldsymbol{M}_{i}=-\boldsymbol{n}_{i} \times \boldsymbol{E}_{i}$, where $\boldsymbol{n}_{i}$ is the normal vector of $S_{i}$ pointing to the inside of the region $\mathrm{D}_{i}$. Because electric and magnetic currents on each interface shall be continuous across the boundary, the electric and magnetic surface currents on $S_{i-1}$ are $\left(\boldsymbol{J}_{i-1}, \boldsymbol{M}_{i-1}\right)$ in region $\mathrm{D}_{i-1}$ and $\left(-\boldsymbol{J}_{i-1},-\boldsymbol{M}_{i-1}\right)$ in region $\mathrm{D}_{i}$. Based on the electric and magnetic field integral equations $[17,21,22]$, the induced electric and magnetic fields $\left(\boldsymbol{E}_{i, i-1}^{\mathrm{JM}}, \boldsymbol{H}_{i, i-1}^{\mathrm{JM}}\right)$ and $\left(\boldsymbol{E}_{i, i}^{\mathrm{JM}}, \boldsymbol{H}_{i, i}^{\mathrm{JM}}\right)$ in region $\mathrm{D}_{i}$ excited by surface current $\left(-\boldsymbol{J}_{i-1},-\boldsymbol{M}_{i-1}\right)$ and $\left(\boldsymbol{J}_{i}, \boldsymbol{M}_{i}\right)$ can be written as

$$
\begin{aligned}
\boldsymbol{E}_{i, i-1}^{\mathrm{M}} & =\boldsymbol{E}_{i}^{-J_{i-1}}+\boldsymbol{E}_{i}^{-M_{i-1}}=\eta_{i} L_{i}\left(-\boldsymbol{J}_{i-1}\right)-K_{i}\left(-\boldsymbol{M}_{i-1}\right), \\
\boldsymbol{E}_{i, i}^{\mathrm{IM}} & =\boldsymbol{E}_{i}^{J_{i}}+\boldsymbol{E}_{i}^{M_{i}}=\eta_{i} L_{i}\left(\boldsymbol{J}_{i}\right)-K_{i}\left(\boldsymbol{M}_{i}\right), \\
\boldsymbol{H}_{i, i-1}^{\mathrm{M}} & =\boldsymbol{H}_{i}^{-J_{i-1}}+\boldsymbol{H}_{i}^{-M_{i-1}}=K_{i}\left(-\boldsymbol{J}_{i-1}\right)+\frac{1}{\eta_{i}} L_{i}\left(-\boldsymbol{M}_{i-1}\right), \\
\boldsymbol{H}_{i, i}^{\mathrm{M}} & =\boldsymbol{H}_{i}^{J_{i}}+\boldsymbol{H}_{i}^{M_{i}}=K_{i}\left(\boldsymbol{J}_{i}\right)+\frac{1}{\eta_{i}} L_{i}\left(\boldsymbol{M}_{i}\right) .
\end{aligned}
$$

The linear operator $L_{i}$ and $K_{i}$ can be defined as

$$
\begin{gathered}
L_{i}(\boldsymbol{F})=-j k_{i} \int_{S_{i}}\left[\boldsymbol{F}+\frac{1}{k_{i}^{2}} \nabla\left(\nabla_{s}^{\prime} \cdot \boldsymbol{F}\right)\right] G_{i} d s^{\prime}, \\
K_{i}(\boldsymbol{F})=\int_{S_{i}} \nabla G_{i} \times \boldsymbol{F} d s^{\prime},
\end{gathered}
$$

where $\nabla_{s}^{\prime}$ is the surface divergence of a vector field. The Green's function in homogeneous isotropic infinite space is given by

$$
G_{i}=G_{i}\left(\boldsymbol{r}, \boldsymbol{r}^{\prime}\right)=\frac{e^{-j k_{i}\left|\boldsymbol{r}-\boldsymbol{r}^{\prime}\right|}}{4 \pi\left|\boldsymbol{r}-\boldsymbol{r}^{\prime}\right|}
$$

where $k_{i}$ is the wave number of region $\mathrm{D}_{i}, r$ is the field point and $r^{\prime}$ is the source point.

According to the definition of the surface currents [19], for a field point $r \in S_{i-1}$ in region $\mathrm{D}_{i}$, the tangential component of electric and magnetic fields can be written as Equations (7)-(10). The left-hand side are induction fields and right-hand side can be expressed in terms of incident fields.

$$
\begin{aligned}
& \left.\left(\boldsymbol{E}_{i}^{-J_{i-1}}+\boldsymbol{E}_{i}^{-M_{i-1}}+\boldsymbol{M}_{i-1} \times \boldsymbol{n}_{i-1}+\boldsymbol{E}_{i}^{J_{i}}+\boldsymbol{E}_{i}^{M_{i}}\right)\right|_{\tan }=-\left.\boldsymbol{E}_{i}^{\text {source }}\right|_{\tan ,}, \quad\left(\boldsymbol{r} \in S_{i-1}\right) \\
& \left.\left(\boldsymbol{H}_{i}^{-J_{i-1}}-\boldsymbol{J}_{i-1} \times \boldsymbol{n}_{i-1}+\boldsymbol{H}_{i}^{-M_{i-1}}+\boldsymbol{H}_{i}^{J_{i}}+\boldsymbol{H}_{i}^{M_{i}}\right)\right|_{\tan }=-\left.\boldsymbol{H}_{i}^{\text {source }}\right|_{\tan , \quad} \quad\left(\boldsymbol{r} \in S_{i-1}\right) .
\end{aligned}
$$

Similarly, for $r \in S_{i}$ in $\mathrm{D}_{i}$,

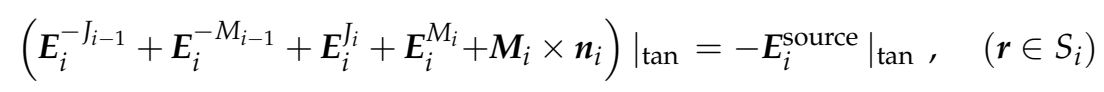




$$
\left.\left(\boldsymbol{H}_{i}^{-J_{i-1}}+\boldsymbol{H}_{i}^{-M_{i-1}}+\boldsymbol{H}_{i}^{J_{i}}-\boldsymbol{J}_{i} \times \boldsymbol{n}_{i}+\boldsymbol{H}_{i}^{M_{i}}\right)\right|_{\tan }=-\left.\boldsymbol{H}_{i}^{\text {source }}\right|_{\tan ,} \quad\left(\boldsymbol{r} \in S_{i}\right) .
$$

For $\boldsymbol{r}=\boldsymbol{r}^{\prime}$, the linear operator $K$ in (5) needs to be split into two parts [22-25]:

$$
K_{i}(\boldsymbol{F})=p . v \cdot \int_{S} \nabla G_{i} \times \boldsymbol{F} d s^{\prime}+\frac{\Omega_{0}}{4 \pi} \boldsymbol{F} \times \boldsymbol{n}=p \cdot v \cdot K_{i}(\boldsymbol{F})+\frac{\Omega_{0}}{4 \pi} \boldsymbol{F} \times \boldsymbol{n},
$$

where p.v. denotes the Cauchy's principal value integration with $\Omega_{0}=2 \pi$ for smooth surface, and $n$ is the outward directed normal vector of the integral surface. By substituting Equation (11) into Equations (7)-(10), we get the electromagnetic fields on the surface in region $\mathrm{D}_{i}$,

$$
\begin{aligned}
& \left.\left(\boldsymbol{E}_{i}^{-J_{i-1}}+\text { p.v. } \boldsymbol{E}_{i}^{-M_{i-1}}+\frac{1}{2} \boldsymbol{M}_{i-1} \times \boldsymbol{n}_{i-1}+\boldsymbol{E}_{i}^{J_{i}}+\boldsymbol{E}_{i}^{M_{i}}\right)\right|_{\tan }=-\left.\boldsymbol{E}_{i}^{\text {source }}\right|_{\tan }, \quad\left(\boldsymbol{r} \in S_{i-1}\right) \\
& \left.\left(\boldsymbol{E}_{i}^{-J_{i-1}}+\boldsymbol{E}_{i}^{-M_{i-1}}+\boldsymbol{E}_{i}^{J_{i}}+\text { p.v. } \boldsymbol{E}_{i}^{M_{i}}+\frac{1}{2} \boldsymbol{M}_{i} \times \boldsymbol{n}_{i}\right)\right|_{\tan }=-\left.\boldsymbol{E}_{i}^{\text {source }}\right|_{\tan , \quad} \quad\left(\boldsymbol{r} \in S_{i}\right)
\end{aligned}
$$

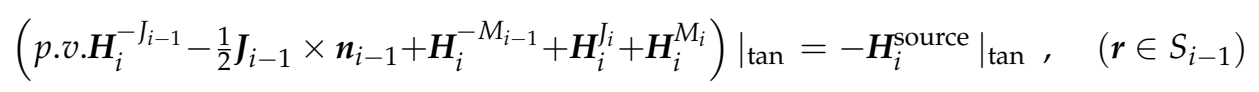

$$
\begin{aligned}
& \left.\left(\boldsymbol{H}_{i}^{-J_{i-1}}+\boldsymbol{H}_{i}^{-M_{i-1}}+\text { p.v. } \boldsymbol{H}_{i}^{J_{i}}-\frac{1}{2} \boldsymbol{J}_{i} \times \boldsymbol{n}_{i}+\boldsymbol{H}_{i}^{M_{i}}\right)\right|_{\tan }=-\left.\boldsymbol{H}_{i}^{\text {source }}\right|_{\tan }, \quad\left(\boldsymbol{r} \in S_{i}\right) .
\end{aligned}
$$

Similarly, we can also calculate the electromagnetic fields on surface $S_{i}$ in region $\mathrm{D}_{i+1}$,

$$
\begin{aligned}
& \left.\left(\boldsymbol{E}_{i+1}^{-J_{i}}+\text { p.v. } \cdot \boldsymbol{E}_{i+1}^{-M_{i}}+\frac{1}{2} \boldsymbol{M}_{i} \times \boldsymbol{n}_{i}+\boldsymbol{E}_{i+1}^{J_{i+1}}+\boldsymbol{E}_{i+1}^{M_{i+1}}\right)\right|_{\tan }=-\left.\boldsymbol{E}_{i+1}^{\text {source }}\right|_{\tan ,} \quad\left(\boldsymbol{r} \in S_{i}\right) \\
& \left.\left(\text { p.v. } \boldsymbol{H}_{i+1}^{-J_{i}}-\frac{1}{2} \boldsymbol{J}_{i} \times \boldsymbol{n}_{i}+\boldsymbol{H}_{i+1}^{-M_{i}}+\boldsymbol{H}_{i+1}^{J_{i+1}}+\boldsymbol{H}_{i+1}^{M_{i+1}}\right)\right|_{\tan }=-\left.\boldsymbol{H}_{i+1}^{\text {source }}\right|_{\tan , \quad} \quad\left(\boldsymbol{r} \in S_{i}\right) .
\end{aligned}
$$

Combining Equations (13) and (16), electric field on surface $S_{i}$ can be written as,

$$
\begin{aligned}
& \left.\left(\boldsymbol{E}_{i}^{-J_{i-1}}+\boldsymbol{E}_{i}^{-M_{i-1}}+\left(\boldsymbol{E}_{i}^{J_{i}}-\boldsymbol{E}_{i+1}^{-J_{i}}\right)+\left(p \cdot v \cdot \boldsymbol{E}_{i}^{M_{i}}-p \cdot v \cdot \boldsymbol{E}_{i+1}^{-M_{i}}\right)-\boldsymbol{E}_{i+1}^{J_{i+1}}-\boldsymbol{E}_{i+1}^{M_{i+1}}\right)\right|_{\tan } \\
& =\left.\left(-\boldsymbol{E}_{i}^{\text {source }}+\boldsymbol{E}_{i+1}^{\text {source }}\right)\right|_{\tan , \quad}\left(\boldsymbol{r} \in S_{i}\right) .
\end{aligned}
$$

The magnetic field on surface $S_{i}$ is obtained by combining Equations (15) and (17),

$$
\begin{aligned}
& \left.\left(\boldsymbol{H}_{i}^{-J_{i-1}}+\boldsymbol{H}_{i}^{-M_{i-1}}+\left(\text { p.v. } \boldsymbol{H}_{i}^{I_{i}}-p \cdot v \cdot \boldsymbol{H}_{i+1}^{-J_{i}}\right)+\left(\boldsymbol{H}_{i}^{M_{i}}-\boldsymbol{H}_{i+1}^{-M_{i}}\right)-\boldsymbol{H}_{i+1}^{I_{i+1}}-\boldsymbol{H}_{i+1}^{M_{i+1}}\right)\right|_{\tan } \\
& =\left.\left(-\boldsymbol{H}_{i}^{\text {source }}+\boldsymbol{H}_{i+1}^{\text {source }}\right)\right|_{\tan ,} \quad\left(\boldsymbol{r} \in S_{i}\right) .
\end{aligned}
$$

\section{Discretization}

For numerical solutions of equivalent electric and magnetic currents on the media interfaces, the Galerkin's method and the Rao-Wilton-Glisson (RWG) basis functions [16,26] are utilized to discretize the continuous formulations Equations (18) and (19) [27]. The media interface of each layer is discretized into planar triangular elements, with $T_{i, n}=T_{i, n}^{+} \cup T_{i, n}^{-}$which is defined as the $n$th triangular element on surface $S_{i}, i=0,1,2, \ldots, K-1$ and $n=0,1,2, \ldots, N_{i}$.

The RWG basis function $f_{i, n}$ assigned to a pair of triangular elements on surface $S_{i}$ is defined as:

$$
f_{i, n}\left(\boldsymbol{r}^{\prime}\right)=\left\{\begin{array}{cl}
\frac{L_{i, n}}{2 A_{i, n}^{+}}\left(\boldsymbol{r}^{\prime}-\boldsymbol{p}_{i, n}^{+}\right), & \boldsymbol{r}^{\prime} \in T_{i, n}^{+} \\
-\frac{L_{i, n}}{2 A_{i, n}^{-}}\left(\boldsymbol{r}^{\prime}-\boldsymbol{p}_{i, n}^{-}\right), & \boldsymbol{r}^{\prime} \in T_{i, n^{\prime}}^{-} \\
0, & \text { otherwise }
\end{array}\right.
$$


where $\boldsymbol{p}_{i, n}^{+}$and $\boldsymbol{p}_{i, n}^{-}$are the vertex of triangular elements $T_{i, n}^{+}$and $T_{i, n^{\prime}}^{-}$which share a common edge of length $L_{i, n}$ [18]. $A_{i, n}^{+}$and $A_{i, n}^{-}$are the areas of triangular elements $T_{i, n}^{+}$and $T_{i, n}^{-}$, respectively. Spatial distribution of RWG functions are shown in Figure 2.

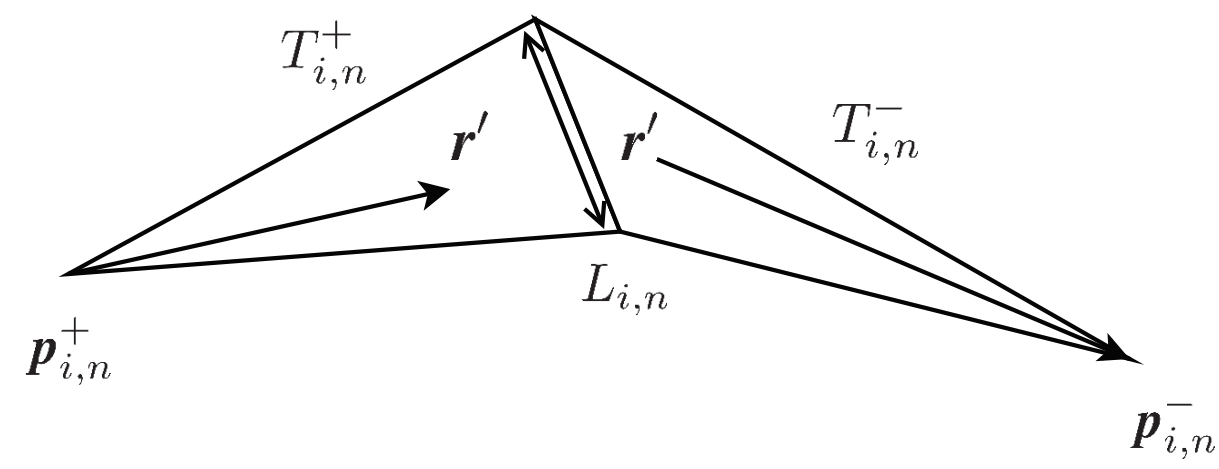

Figure 2. Spatial distribution of Rao-Wilton-Glisson (RWG) functions.

The unknown surface current distribution $\boldsymbol{J}_{i}$ and $\boldsymbol{M}_{i}$ on surface $S_{i}$ are replaced by an expansion in the basis in numerical computations:

$$
\boldsymbol{J}_{i}=\sum_{n=1}^{N_{i}} \alpha_{i, n} f_{i, n}, \boldsymbol{M}_{i}=\sum_{n=1}^{N_{i}} \beta_{i, n} f_{i, n}
$$

where $\alpha_{i, n}$ and $\beta_{i, n}\left(i=0,1,2, \ldots, K-1 ; n=0,1,2, \ldots, N_{i}\right)$ are the $n$th complex expansion coefficients of $\boldsymbol{J}_{i}$ and $\boldsymbol{M}_{i}$. By substituting (21) into (18) and (19), and testing them with RWG basis, the matrix equation for media interface $S_{i}$ can be written as

$$
\left[\begin{array}{lll}
\mathbf{Z}_{i-1}^{i} & \mathbf{Z}_{i}^{i} \mathbf{Z}_{i+1}^{i}
\end{array}\right]\left[\begin{array}{c}
\mathbf{I}_{i-1} \\
\mathbf{I}_{i} \\
\mathbf{I}_{i+1}
\end{array}\right]=\mathbf{V}_{i}^{\mathrm{EH}}
$$

where,

$$
\begin{aligned}
& \mathbf{Z}_{i-1}^{i}=\left(\begin{array}{ll}
{\left[Z_{E, m n}^{J_{i-1}}\right]_{N_{i} \times N_{i-1}}} & {\left[Z_{E, m n}^{M_{i-1}}\right]_{N_{i} \times N_{i-1}}} \\
{\left[Z_{H, m n}^{J_{i-1}}\right]_{N_{i} \times N_{i-1}}} & {\left[Z_{H, m n}^{M_{i-1}}\right]_{N_{i} \times N_{i-1}}}
\end{array}\right),
\end{aligned}
$$

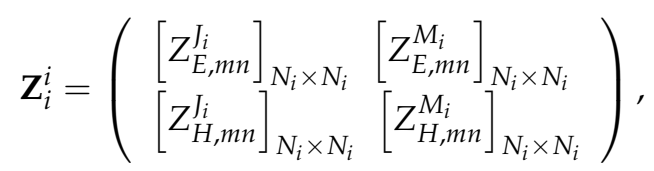

$$
\begin{aligned}
& \mathbf{Z}_{i+1}^{i}=\left(\begin{array}{ll}
{\left[Z_{E, m n}^{J_{i+1}}\right]_{N_{i} \times N_{i+1}}} & {\left[Z_{E, m n}^{M_{i+1}}\right]_{N_{i} \times N_{i+1}}} \\
{\left[Z_{H, m n}^{J_{i+1}}\right]_{N_{i} \times N_{i+1}}} & {\left[Z_{H, m n}^{M_{i+1}}\right]_{N_{i} \times N_{i+1}}}
\end{array}\right), \\
& {\left[\begin{array}{c}
\mathbf{I}_{i-1} \\
\mathbf{I}_{i} \\
\mathbf{I}_{i+1}
\end{array}\right]=\left(\begin{array}{l}
{\left[\alpha_{i-1, n}\right]_{N_{i-1} \times 1}} \\
{\left[\beta_{i-1, n}\right]_{N_{i-1} \times 1}} \\
{\left[\alpha_{i, n}\right]_{N_{i} \times 1}} \\
{\left[\beta_{i, n}\right]_{N_{i} \times 1}} \\
{\left[\alpha_{i+1, n}\right]_{N_{i+1} \times 1}} \\
{\left[\beta_{i+1, n}\right]_{N_{i+1} \times 1}}
\end{array}\right),}
\end{aligned}
$$


Apple. Sci. 2017, 7, 74

6 of 15

$$
\mathbf{V}_{i}^{\mathrm{EH}}=\left(\begin{array}{c}
{\left[-V_{i, m}^{E i}+V_{i+1, m}^{E i}\right]_{N_{i} \times 1}} \\
{\left[-V_{i, m}^{H i}+V_{i+1, m}^{H i}\right]_{N_{i} \times 1}}
\end{array}\right) .
$$

The submatrix in the matrix equation can be expressed as:

$$
\begin{aligned}
& Z_{E, m n}^{J_{i-1}}=\int_{T_{i, m}} f_{i, m}(\boldsymbol{r}) \cdot \eta_{i} L_{i}\left(-f_{i-1, n}\left(\boldsymbol{r}^{\prime}\right)\right) d s, \\
& Z_{E, m n}^{M_{i-1}}=-\int_{T_{i, m}} f_{i, m}(\boldsymbol{r}) \cdot K_{i}\left(-f_{i-1, n}\left(\boldsymbol{r}^{\prime}\right)\right) d s, \\
& Z_{E, m n}^{J_{i}}=\int_{T_{i, m}} f_{i, m}(\boldsymbol{r}) \cdot \eta_{i} L_{i}\left(f_{i, n}\left(\boldsymbol{r}^{\prime}\right)\right) d s-\int_{T_{i, m}} f_{i, m}(\boldsymbol{r}) \cdot \eta_{i+1} L_{i+1}\left(-f_{i, n}\left(\boldsymbol{r}^{\prime}\right)\right) d s, \\
& Z_{E, m n}^{M_{i}}=-\int_{T_{i, m}} f_{i, m}(\boldsymbol{r}) \cdot p \cdot v \cdot K_{i}\left(f_{i, n}\left(\boldsymbol{r}^{\prime}\right)\right) d s+\int_{T_{i, m}} f_{i, m}(\boldsymbol{r}) \cdot p \cdot v \cdot K_{i+1}\left(-f_{i, n}\left(\boldsymbol{r}^{\prime}\right)\right) d s, \\
& Z_{E, m n}^{J_{i+1}}=-\int_{T_{i, m}} f_{i, m}(\boldsymbol{r}) \cdot \eta_{i+1} L_{i+1}\left(f_{i+1, n}\left(\boldsymbol{r}^{\prime}\right)\right) d s, \\
& Z_{E, m n}^{M_{i+1}}=\int_{T_{i, m}} f_{i, m}(\boldsymbol{r}) \cdot K_{i+1}\left(f_{i+1, n}\left(\boldsymbol{r}^{\prime}\right)\right) d s, \\
& Z_{H, m n}^{J_{i-1}}=\int_{T_{i, m}} f_{i, m}(\boldsymbol{r}) \cdot K_{i}\left(-f_{i-1, n}\left(\boldsymbol{r}^{\prime}\right)\right) d s, \\
& Z_{H, m n}^{M_{i-1}}=\int_{T_{i, m}} f_{i, m}(\boldsymbol{r}) \cdot \frac{1}{\eta_{i}} L_{i}\left(-f_{i-1, n}\left(\boldsymbol{r}^{\prime}\right)\right) d s, \\
& Z_{H, m n}^{J_{i}}=\int_{T_{i, m}} f_{i, m}(\boldsymbol{r}) \cdot p \cdot v \cdot K_{i}\left(f_{i, n}\left(\boldsymbol{r}^{\prime}\right)\right) d s-\int_{T_{i, m}} f_{i, m}(\boldsymbol{r}) \cdot p \cdot v \cdot K_{i+1}\left(-f_{i, n}\left(\boldsymbol{r}^{\prime}\right)\right) d s, \\
& Z_{H, m n}^{M_{i}}=\int_{T_{i, m}} f_{i, m}(\boldsymbol{r}) \cdot \frac{1}{\eta_{i}} L_{i}\left(f_{i, n}\left(\boldsymbol{r}^{\prime}\right)\right) d s-\int_{T_{i, m}} f_{i, m}(\boldsymbol{r}) \cdot \frac{1}{\eta_{i+1}} L_{i+1}\left(-f_{i, n}\left(\boldsymbol{r}^{\prime}\right)\right) d s, \\
& Z_{H, m n}^{J_{i+1}}=-\int_{T_{i, m}} f_{i, m}(\boldsymbol{r}) \cdot K_{i+1}\left(f_{i+1, n}\left(\boldsymbol{r}^{\prime}\right)\right) d s, \\
& Z_{H, m n}^{M_{i+1}}=-\int_{T_{i, m}} f_{i, m}(\boldsymbol{r}) \cdot \frac{1}{\eta_{i+1}} L_{i+1}\left(f_{i+1, n}\left(\boldsymbol{r}^{\prime}\right)\right) d s .
\end{aligned}
$$

The basis $f_{j, n}\left(\boldsymbol{r}^{\prime}\right),(j=0,1,2, \ldots, K-1)$ in medium $\mathrm{D}_{i}$ is operated by the linear operator $L$ and $K$ :

$$
\begin{gathered}
L_{i}\left(\boldsymbol{f}_{j, n}\left(\boldsymbol{r}^{\prime}\right)\right)=-j k_{i} \int_{T_{j, n}}\left[\left(\boldsymbol{f}_{j, n}\left(\boldsymbol{r}^{\prime}\right)\right)+\frac{1}{k_{i}^{2}} \nabla\left(\nabla_{s}^{\prime} \cdot\left(\boldsymbol{f}_{j, n}\left(\boldsymbol{r}^{\prime}\right)\right)\right)\right] G_{i}\left(\boldsymbol{r}, \boldsymbol{r}^{\prime}\right) d s^{\prime}, \\
K_{i}\left(\boldsymbol{f}_{j, n}\left(\boldsymbol{r}^{\prime}\right)\right)=\int_{T_{j, n}} \nabla G_{i}\left(\boldsymbol{r}, \boldsymbol{r}^{\prime}\right) \times \boldsymbol{f}_{j, n}\left(\boldsymbol{r}^{\prime}\right) d s^{\prime} .
\end{gathered}
$$

For $r \in S_{i}$, the electric and magnetic excitation source matrix in $\mathrm{D}_{i}$ can be expressed as: 


$$
\begin{gathered}
V_{i, m}^{E i}=\int_{T_{i, m}} f_{i, m}(\boldsymbol{r}) \cdot \boldsymbol{E}_{i}^{\text {source }} d s, \quad V_{i+1, m}^{E i}=\int_{T_{i, m}} f_{i, m}(\boldsymbol{r}) \cdot \boldsymbol{E}_{i+1}^{\text {source }} d s, \\
V_{i, m}^{H i}=\int_{T_{i, m}} f_{i, m}(\boldsymbol{r}) \cdot \boldsymbol{H}_{i}^{\text {source }} d s, \quad V_{i+1, m}^{H i}=\int_{T_{i, m}} f_{i, m}(\boldsymbol{r}) \cdot \boldsymbol{H}_{i+1}^{\text {source }} d s .
\end{gathered}
$$

According to Equation (22), the matrix equation for all surfaces with block tridiagonal matrix equation is derived as follows:

$$
\left(\begin{array}{ccccccc}
\mathbf{Z}_{0}^{0} & \mathbf{Z}_{1}^{0} & \mathbf{0} & & & & \\
\mathbf{Z}_{0}^{1} & \mathbf{Z}_{1}^{1} & \mathbf{Z}_{2}^{1} & \mathbf{0} & & & \\
\mathbf{0} & \mathbf{Z}_{1}^{2} & \mathbf{Z}_{2}^{2} & \mathbf{Z}_{3}^{2} & \mathbf{0} & & \\
& & & \ddots & & & \\
& & \mathbf{0} & \mathbf{Z}_{K-4}^{K-3} & \mathbf{Z}_{K-3}^{K-3} & \mathbf{Z}_{K-2}^{K-3} & \mathbf{0} \\
& & & \mathbf{0} & \mathbf{Z}_{K-3}^{K-2} & \mathbf{Z}_{K-2}^{K-2} & \mathbf{Z}_{K-1}^{K-2} \\
& & & & \mathbf{0} & \mathbf{Z}_{K-2}^{K-1} & \mathbf{Z}_{K-1}^{K-1}
\end{array}\right)\left(\begin{array}{c}
\mathbf{I}_{0} \\
\mathbf{I}_{1} \\
\mathbf{I}_{2} \\
\mathbf{I}_{3} \\
\vdots \\
\mathbf{I}_{K-2} \\
\mathbf{I}_{K-1}
\end{array}\right)=\left(\begin{array}{c}
\mathbf{V}_{0}^{\mathrm{EH}} \\
\mathbf{V}_{1}^{\mathrm{EH}} \\
\mathbf{V}_{2}^{\mathrm{EH}} \\
\vdots \\
\mathbf{V}_{K-3}^{\mathrm{EH}} \\
\mathbf{V}_{K-2}^{\mathrm{EH}} \\
\mathbf{V}_{K-1}^{\mathrm{EH}}
\end{array}\right) .
$$

Equation (39) is the relation between the surface current, impedance matrix and incidence fields in $K$-layered dissipative medium structures. The impedance matrix is a square matrix with the number of elements equal to $\sum_{i=0}^{K-1} 2 N_{i} \times \sum_{i=0}^{K-1} 2 N_{i}$, with $\sum_{i=0}^{K-1}\left(2 N_{i}\right)\left(2 N_{i-1}+2 N_{i}+2 N_{i+1}\right)$ non-zero data in the impedance matrix. Both the number of unknown surface current coefficients and excitation source matrix are $\sum_{i=0}^{K-1} 2 N_{i} \times 1$. Therefore, the surface current coefficients can be obtained by solving the matrix equation.

\section{Numerical Examples}

In this section, three experiments are constructed to analyze the performance of the proposed method with the vertical or horizontal dipole buried in media including sea, wet and other dissipative materials. The algorithm is implemented by MATLAB $2011 \mathrm{~b}$ on a computer with CPU i3-4030U working at $1.9 \mathrm{GHz}$ with $4 \mathrm{~GB}$ RAM and a Windows 10 operating system. The Low Frequency (LF) Domain Solver of CST Studio Suite in the same computer is used to simulate the models for comparison.

We first consider a non-planar air-sea-seabed structure with a hemispherical depression at the seabed shown in Figure 3 for the application that the naval vessel receives signals from the transmitter burried in the seabed. The air permittivity and permeability are $\varepsilon_{0} \mu_{0}$, respectively, which are the same as vacuum. Generally, the conductivity of the sea is $\sigma_{1}=4 \mathrm{~S} / \mathrm{m}$, and the permittivity and permeability of the sea are $\varepsilon_{1}=74 \varepsilon_{0}$ and $\mu_{1}=\mu_{0}$, respectively. Conductivity of the seabed is $\sigma_{2}=0.01 \mathrm{~S} / \mathrm{m}$, with permittivity $\varepsilon_{2}=13 \varepsilon_{0}$ and permeability $\mu_{2}=\mu_{0}$. Media interface $S_{0}$ and $S_{1}$ are both circular with radius $r=50 \mathrm{~m}$, located at plane $z=0 \mathrm{~m}$ and $z=-20 \mathrm{~m}$, respectively. The hemispherical depression is set at point $(0,0,-20)$ with radius $r=10 \mathrm{~m}$. A vertical electric dipole excitation source is located at point $(0,0,-25)$, with dipole moment $1 \mathrm{~A} \cdot \mathrm{m}$ and the frequency is $100 \mathrm{~Hz}$. In order to reduce the mesh amount, we refine the mesh in the source areas and other part is sparse. The number of triangles on the air-sea interface $S_{0}$ and the sea-seabed interface $S_{1}$ are 856 and 1528. As a result, 7088 unknowns are generated. In numerical calculations, the processor takes $49.4 \mathrm{~s}$ and $0.58 \mathrm{~GB}$ to solve the matrix equation. However, the calculation of the same model with 1, 046, 366 unknowns using CST takes $156 \mathrm{~s}$ and 2.3 GB of memory. 


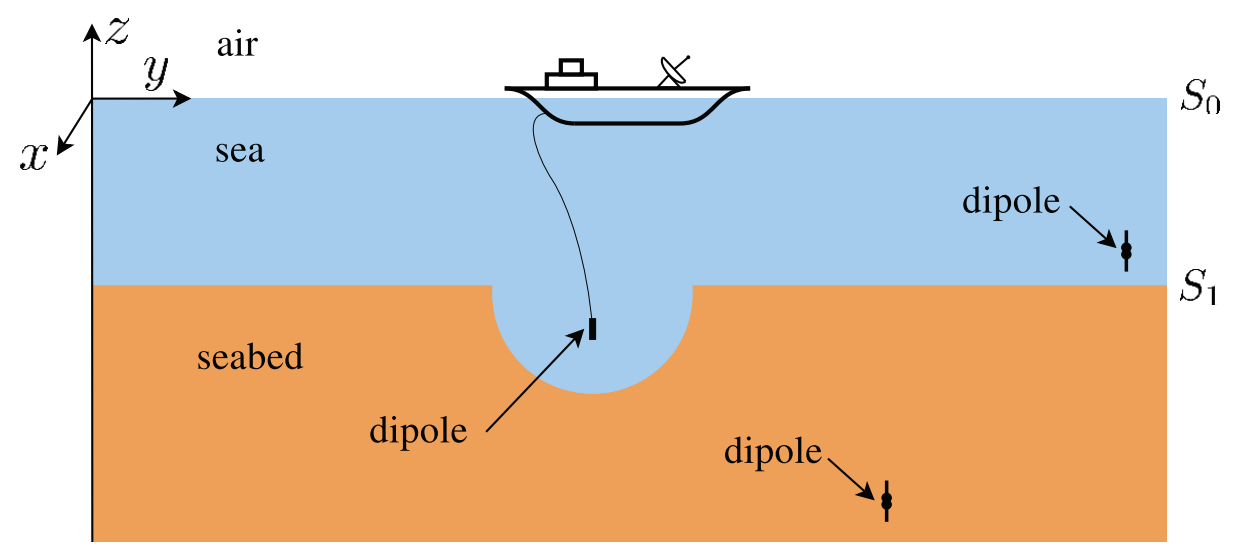

Figure 3. Air-sea-seabed layered media structure with a non-planar surface.

Figure 4 shows the $x$-direction electric field and the total electric field at point $(x, 0,-60)$, $0 \leq x \leq 25$. The results calculated by CST are also plotted in Figure 4 for comparison. These results show clearly that the numerical results calculated by the proposed method are close to the results computed by CST, with most errors within a range of $\pm 2 \%$. However, from the results listed in Table 1 , it can be observed that there are relatively large errors in the first and last column, which are larger than $2 \%$. As the electric field in $E_{x}$ close to $z$-axis is a minimum value, non-uniform discretized mesh can introduce a relatively large error to the first column in numerical calculations. The sparse mesh far from source also causes large error in the last column. It can be also observed from the results in Table 2 that most of the results are within the error range of $\pm 2 \%$. However, there is relatively large error when field point is in the sparse mesh area. The comparisons indicate that the proposed method provides good performance in solving time and high accuracy results for the electric dipole in the two-layered dissipative structure.

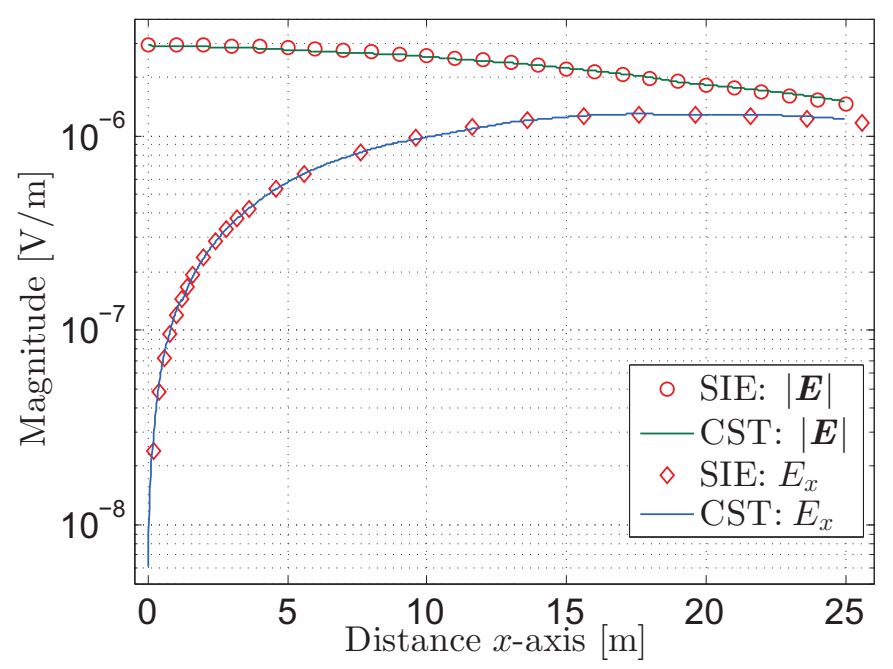

Figure 4. The total electric field and the electric field in $x$-direction at test point $(x, 0,-60)$. SIE: surface integral equation. CST: Computer Simulation Technology. 
Table 1. Electric field in $x$-direction component at test point $(x, 0,-60)$.

\begin{tabular}{cccccc}
\hline$x$-Axis $(\mathbf{m})$ & $\mathbf{1}$ & $\mathbf{2}$ & $\mathbf{1 3 . 6}$ & $\mathbf{1 9 . 6}$ & $\mathbf{2 3 . 6}$ \\
\hline $\mathrm{CST}(\mathrm{dB} \mu \mathrm{V} / \mathrm{m})$ & -18.06 & -12.43 & 1.57 & 2.12 & 1.98 \\
$\mathrm{SIE}(\mathrm{dB} \mu \mathrm{V} / \mathrm{m})$ & -18.42 & -12.43 & 1.58 & 2.14 & 1.73 \\
Error $\%$ & -4.0 & 0 & 0.2 & 0.3 & -2.9 \\
\hline
\end{tabular}

Table 2. The total electric field at test point $(x, 0,-60)$.

\begin{tabular}{cccccc}
\hline $\boldsymbol{x}$-Axis (m) & $\mathbf{0}$ & $\mathbf{2}$ & $\mathbf{1 3}$ & $\mathbf{2 0}$ & $\mathbf{2 5}$ \\
\hline $\mathrm{CST}(\mathrm{dB} \mu \mathrm{V} / \mathrm{m})$ & 9.28 & 9.16 & 7.49 & 5.25 & 3.52 \\
$\mathrm{SIE}(\mathrm{dB} \mu \mathrm{V} / \mathrm{m})$ & 9.37 & 9.34 & 7.49 & 5.25 & 3.35 \\
Error $\%$ & 1.0 & 2.1 & 0 & 0 & -2.0 \\
\hline
\end{tabular}

For the second example, we consider the air-rock-earth-mine layered structure shown in Figure 5. It can be used in Through-The-Earth communication for mines, where communication devices communicate with other nodes by using horizontal electric dipoles located in the earth layer. The frequency of excitation source is $10 \mathrm{kHz}$. The conductivity of the rock layer is $\sigma_{1}=0.001 \mathrm{~S} / \mathrm{m}$, and the permittivity and permeability of the rock are $\varepsilon_{1}=3 \varepsilon_{0}$ and $\mu_{1}=\mu_{0}$, respectively. Conductivity of the earth layer is $\sigma_{2}=0.01 \mathrm{~S} / \mathrm{m}$, with permittivity $\varepsilon_{2}=4 \varepsilon_{0}$ and permeability $\mu_{2}=\mu_{0}$. The conductivity of the mine layer is $\sigma_{3}=0.001 \mathrm{~S} / \mathrm{m}$, and the permittivity and permeability of the mine are $\varepsilon_{3}=3 \varepsilon_{0}$ and $\mu_{3}=\mu_{0}$, respectively. Media interfaces $S_{0}, S_{1}$ and $S_{2}$ are circular of radius $r=1000 \mathrm{~m}$. Media interfaces $S_{0}, S_{1}$ and $S_{2}$ are located at plane $z=0 \mathrm{~m}, z=-150 \mathrm{~m}$ and $z=-350 \mathrm{~m}$. The $x$-directional horizontal electric dipole with dipole moment $1 \mathrm{~A} \cdot \mathrm{m}$ is located at $(0,0,-250)$.

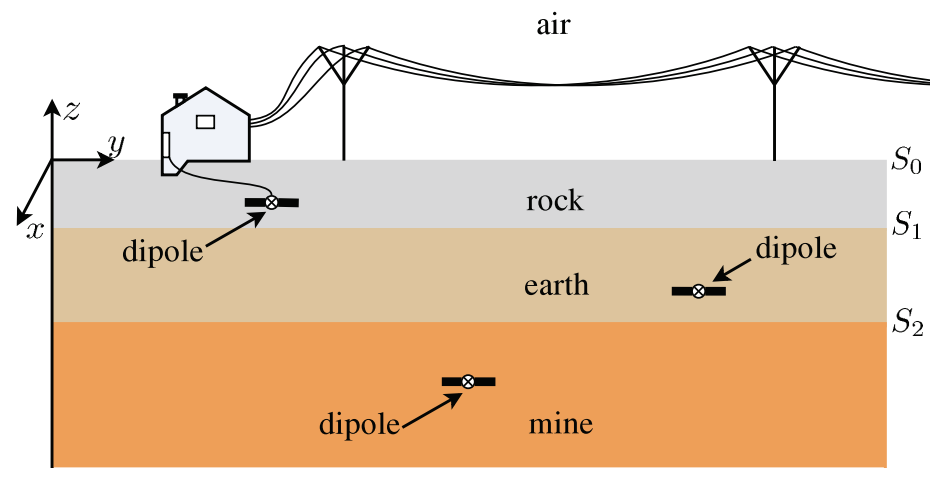

Figure 5. Air-rock-earth-mine layered media structure.

The $x$-component electric field propagation at point $(0, y,-500), 0 \leq y \leq 1000$ is plotted in Figure 6 . The $z$-component electric field propagation and the $y$-component magnetic field propagation at point $(x, 0,-500), 0 \leq x \leq 1000$ are plotted in Figures 7 and 8 , respectively. We can notice that the numerical results calculated by the proposed method are close to the results computed by CST. It can be observed from Tables $3-5$ that most of the numerical results are within the error range $\pm 3 \%$. The mesh is sparse at the boundary area, which results in relatively large deviation when the test point gets closer to the boundary, and the magnetic field results are more accurate than electric field results.

Each of the interfaces $S_{0}, S_{1}$ and $S_{2}$ is meshed into 710 triangle patches, resulting in 6288 unknowns. It takes $37.9 \mathrm{~s}$ and $0.36 \mathrm{~GB}$ to solve the matrix equation by the use of the proposed method. However, the calculation of the same model with 461,970 unknowns using CST takes $55 \mathrm{~s}$ and 1.7 GB memory. 


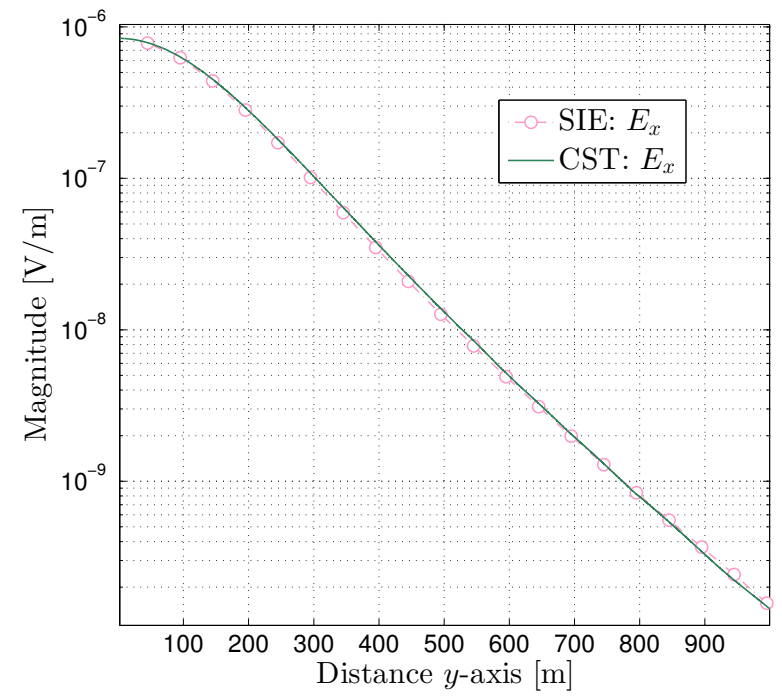

Figure 6. Electric field in the $x$-direction component at test point $(0, y,-500)$.

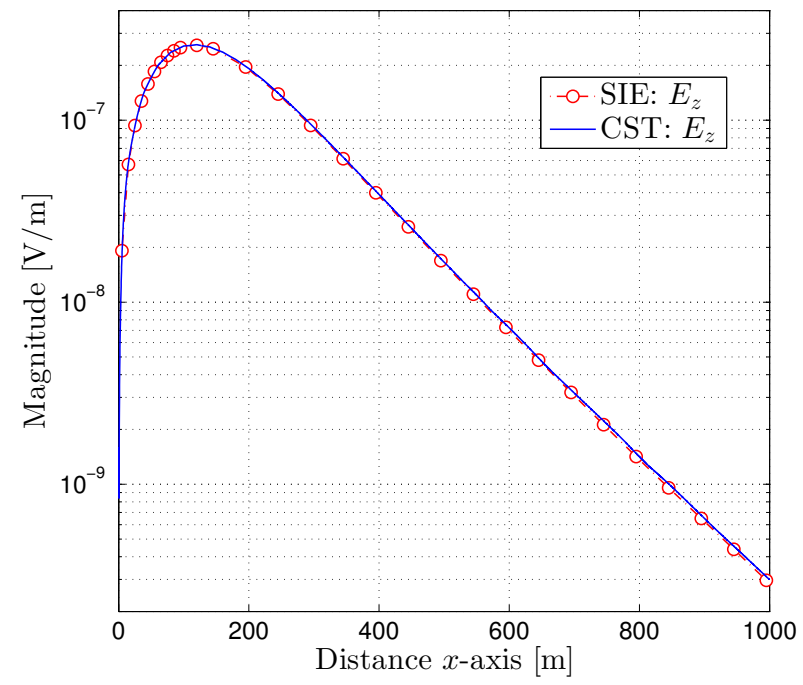

Figure 7. Electric field in the $z$-direction component at test point $(x, 0,-500)$. 


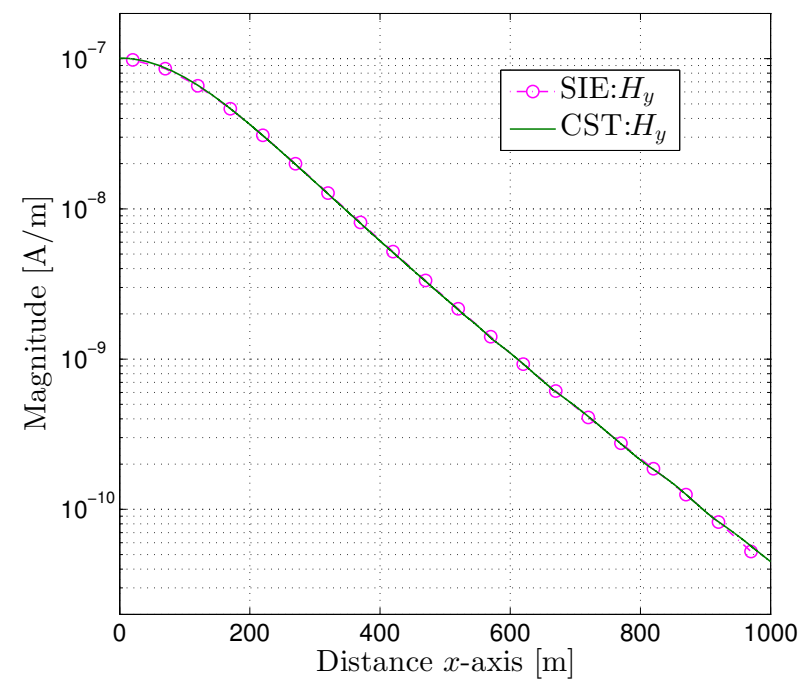

Figure 8. Magnetic field in the $y$-direction component at test point $(x, 0,-500)$.

Table 3. Electric field in the $x$-direction component at test point $(0, y,-500)$.

\begin{tabular}{cccccc}
\hline y-Axis $(\mathbf{m})$ & $\mathbf{4 5}$ & $\mathbf{9 5}$ & $\mathbf{6 9 5}$ & $\mathbf{7 4 5}$ & $\mathbf{8 4 5}$ \\
\hline $\mathrm{CST}(\mathrm{dB} \mathrm{V} / \mathrm{m})$ & -122.09 & -123.97 & -173.81 & -178.06 & -185.37 \\
$\mathrm{SIE}(\mathrm{dB} \mathrm{V} / \mathrm{m})$ & -122.13 & -124.10 & -174.02 & -177.79 & -185.13 \\
Error \% & -0.51 & -1.4 & -2.4 & 3.2 & 2.7 \\
\hline
\end{tabular}

Table 4. Electric field in the $z$-direction component at test point $(x, 0,-500)$.

\begin{tabular}{cccccc}
\hline$x$-Axis (m) & 45 & 95 & 695 & 745 & 845 \\
\hline $\mathrm{CST}(\mathrm{dB} \mathrm{V} / \mathrm{m})$ & -136.03 & -132.04 & -169.63 & -173.03 & -180.00 \\
$\mathrm{SIE}(\mathrm{dB} \mathrm{V} / \mathrm{m})$ & -136.03 & -132.04 & -169.89 & -173.43 & -180.40 \\
Error $\%$ & 0.0 & 0.0 & -3.0 & -4.4 & -4.5 \\
\hline
\end{tabular}

Table 5. Magnetic field in the $y$-direction component at test point $(x, 0,-500)$.

\begin{tabular}{cccccc}
\hline$x$-Axis (m) & $\mathbf{7 0}$ & $\mathbf{1 7 0}$ & $\mathbf{7 2 0}$ & $\mathbf{7 7 0}$ & $\mathbf{8 7 0}$ \\
\hline $\mathrm{CST}(\mathrm{dB} \mathrm{A} / \mathrm{m})$ & -141.25 & -146.66 & -187.65 & -191.27 & -198.01 \\
$\mathrm{SIE}(\mathrm{dB} \mathrm{A} / \mathrm{m})$ & -141.34 & -146.67 & -187.76 & -191.20 & -198.05 \\
Error \% & -1.1 & 0.1 & -1.3 & 0.8 & -0.4 \\
\hline
\end{tabular}

The proposed method is used to simulate the electromagnetic fields excited by horizontal electric dipole in a dissipative sphere, which is a closed structure coated by layered materials and is shown in Figure 9. The external sphere is located at origin and has a radius of $6 \mathrm{~m}$ with conductivity $\sigma_{1}=0.001 \mathrm{~S} / \mathrm{m}$, permittivity $\varepsilon_{1}=3.1 \varepsilon_{0}$ and permeability $\mu_{1}=\mu_{0}$. The radius of the middle sphere is $3 \mathrm{~m}$ with conductivity $\sigma_{2}=0.01 \mathrm{~S} / \mathrm{m}$, permittivity $\varepsilon_{2}=2 \varepsilon_{0}$ and permeability $\mu_{2}=\mu_{0}$, which is located at point $(-1,0,0)$. The internal sphere is located at origin and has a radius of $1 \mathrm{~m}$, where the conductivity is $\sigma_{3}=0.1 \mathrm{~S} / \mathrm{m}$, permittivity is $\varepsilon_{3}=2 \varepsilon_{0}$, and permeability is $\mu_{3}=\mu_{0}$. A horizontal electric dipole excitation source is located at origin with dipole moment of $1 \mathrm{~A} \cdot \mathrm{m}$ along $x$-axis, and 
the frequency is $1 \mathrm{MHz}$. After discretization, the numbers of triangles on the external sphere $S_{0}$, the middle sphere $S_{1}$ and the internal sphere $S_{2}$ are 1204, 604 and 626, respectively. As a result, 7302 unknowns are generated. It takes $54.3 \mathrm{~s}$ and $0.66 \mathrm{~GB}$ of memory to solve the matrix equation in numerical calculations. Compared with the proposed method, the calculation of the same model with 923, 446 unknowns using CST takes $150 \mathrm{~s}$ and $1.9 \mathrm{~GB}$ of memory, which shows that the proposed method has a good performance in solving time and storage space.

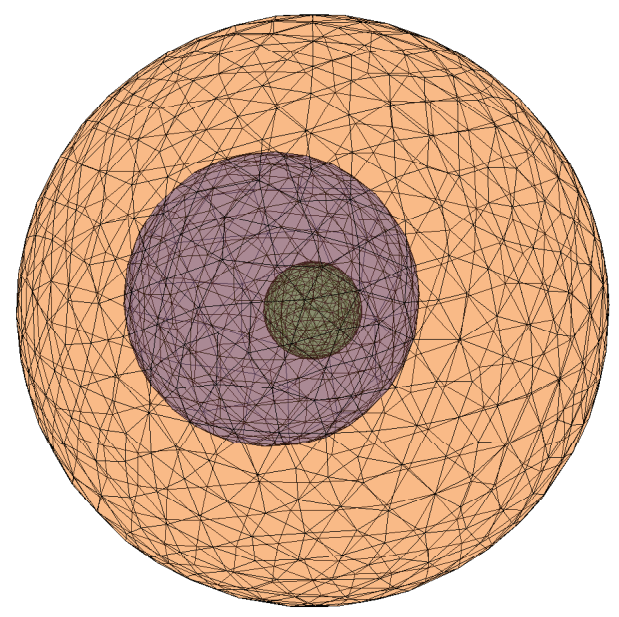

Figure 9. Horizontal electric dipole in sphere structure. External sphere: Yellow. Middle sphere: Purple. Internal sphere: Green.

Figure 10 shows the near magnetic field component $H_{z}$ as a function of the observation angle in plane $z=0$, and Figure 11 illustrates the near electric field component $E_{y}$, which are compared with CST. We can observe that the numerical results obtained from the proposed method are in good agreement with the results obtained by CST. Figure 12 gives the root-mean-square (RMS) error of different observation radius. The Root-mean-square (RMS) error is given by [28]:

$$
\operatorname{RMS}(x)=\sqrt{\frac{\sum_{i=1}^{N}\left|x^{\operatorname{SIE}}(\boldsymbol{r})-x^{\mathrm{CST}}(\boldsymbol{r})\right|^{2}}{\sum_{i=1}^{N}|x \operatorname{CST}(\boldsymbol{r})|^{2}}}
$$

where $x^{\mathrm{SIE}}$ is the calculated quantity and $x^{\mathrm{CST}}$ refers to the corresponding CST result. We can see that the $H_{z}$ RMS error is smaller than that of $E_{y}$ of each observation radius. It indicates that the proposed method has a better performance in solving magnetic field than solving electric field. In the CST model, the excitation source is a quasi-ideal horizontal electric dipole. As a result, the comparison model is different from our simulation model, and the RMS error seems to be higher when the observation point is close to the source. When the observation radius are larger than $0.6 \mathrm{~m}$, the numerical results are still in good agreement with the comparison model with RMS error of less than $2 \%$ for magnetic fields and $4 \%$ for electric fields. 


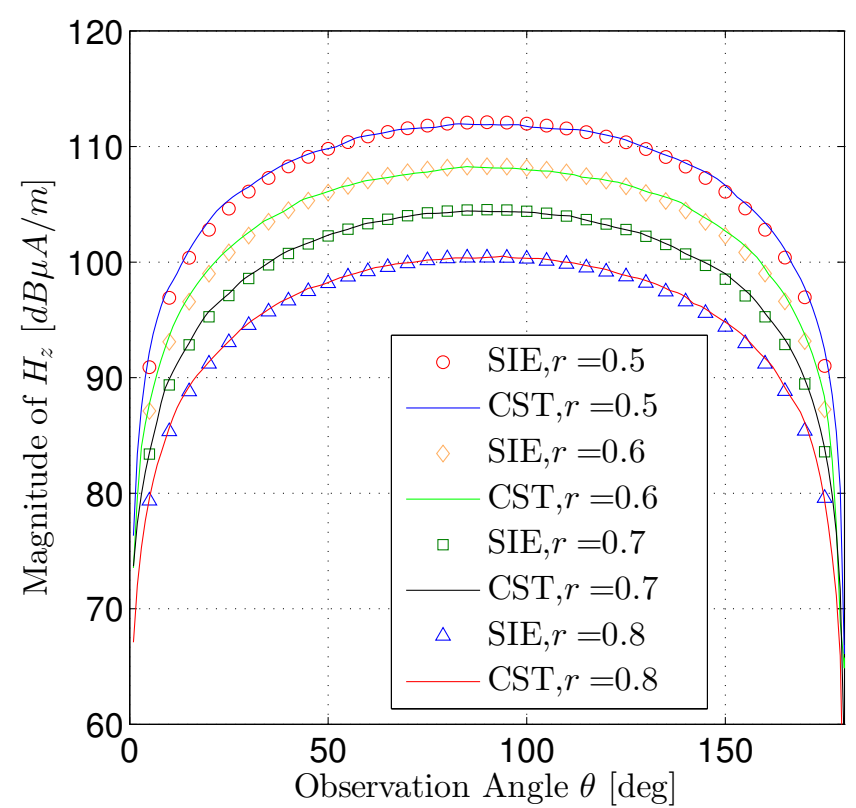

Figure 10. The magnetic fields $H_{z}$ for observation radius $r=0.5,0.6,0.7$ and $0.8 \mathrm{~m}$.

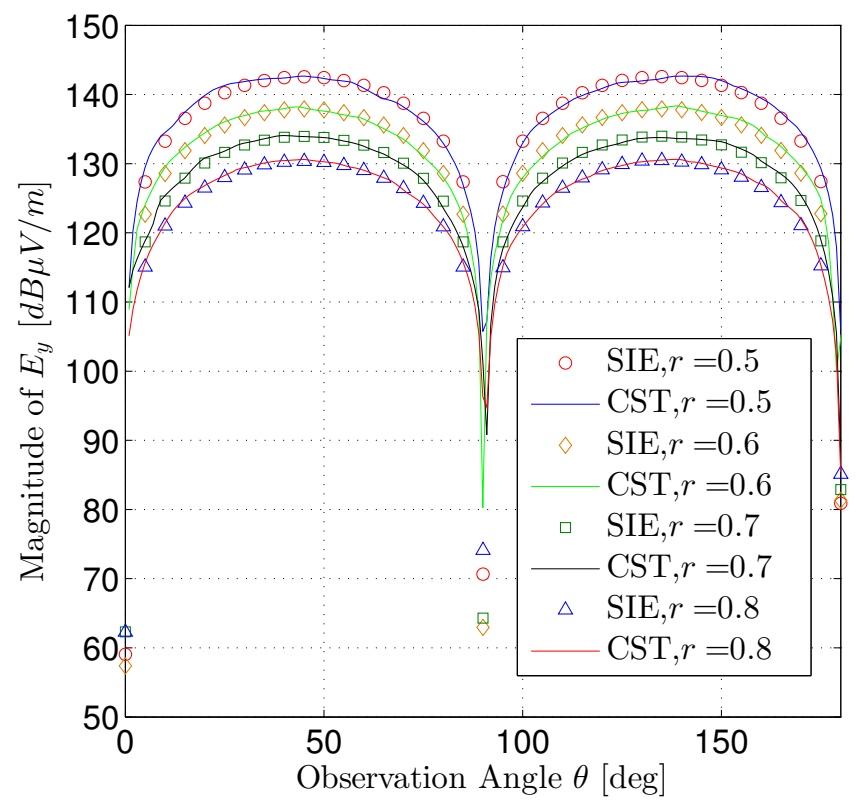

Figure 11. The electric fields $E_{y}$ for observation radius $r=0.5,0.6,0.7$ and $0.8 \mathrm{~m}$. 


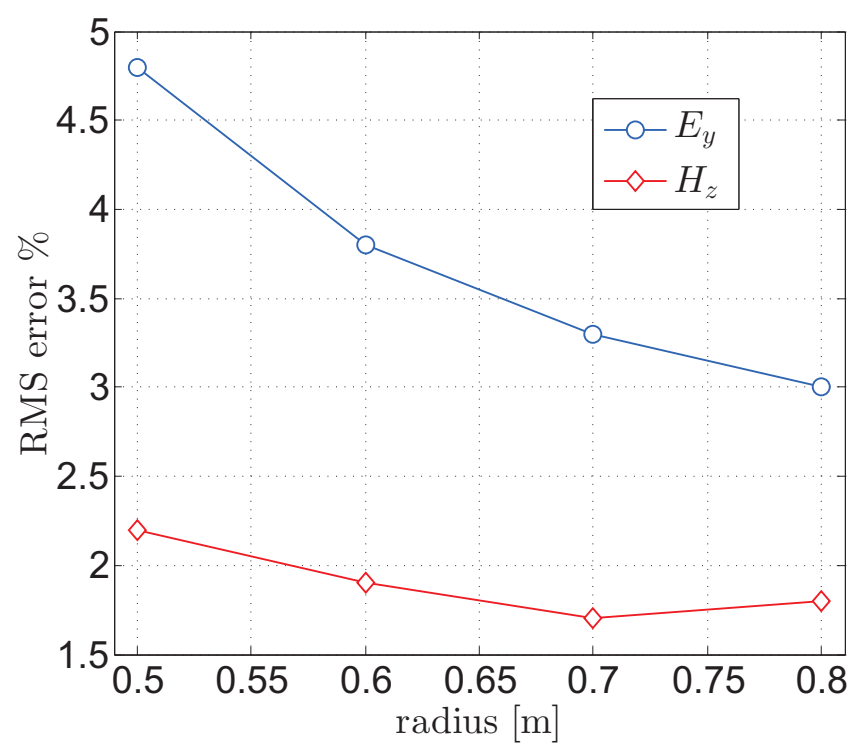

Figure 12. Root-mean-square (RMS) errors for $H_{z}$ and $E_{y}$ with different observation radius.

\section{Conclusions}

In this paper, a novel method based on PMCHWT integral equations has been proposed to study the electromagnetic fields excited by the vertical or horizontal electric dipole in a layered dissipative medium region. The electromagnetic fields in each layer are excited only by the equivalent surface current at the top/bottom of the layer and the dipole in it. A block tridiagonal matrix system is derived, which results in a much more straightforward treatment to handle non-planar layered structure, multilayered planar structure and closed structure coated with arbitrary layered dissipative materials. To analyze the performance of the method, non-planar air-sea-seabed, air-rock-earth-mine and multilayered sphere structures are investigated. The numerical results show that accurate near fields in the layered dissipative medium region are obtained by the method with deviation of $2 \%$. The proposed method can give a better performance with respect to the solving time and storage space in comparison with the CST in handling near-filled problems. Other areas for future work include filling and solving the block tridiagonal matrix using fast/parallel techniques.

Acknowledgments: This research has been supported by the National Key Research and Development Program of China-Government Corporation Special Program (2016YFE0111100), Fundamental Research Funds for the Central Universities (GK2080260160 and GK2080260166).

Author Contributions: Yidong $\mathrm{Xu}$ did the mathematical modeling and the simulations. He also wrote the draft of the paper. Wei Xue and Yingsong Li put forward to the idea and checked the simulation of this paper. Lili Guo and Wenjing Shang contributed to the revisions and the discussion of the results.

Conflicts of Interest: The authors declare no conflict of interest.

\section{References}

1. Kraichman, M.B. Handbook of Electromagnetic Propagation in Conducting Media; Headquarters Naval Material Command; U.S. Government Publishing Office: Washington, DC, USA, 1970.

2. King, R.W.; Owens, M.; Wu, T.T. Lateral Electromagnetic Waves: Theory and Applications To Communications, Geophysical Exploration, and Remote Sensing; Springer Science \& Business Media: Berlin, Germany, 2012.

3. Collin, R.E. Some observations about the near zone electric field of a Hertzian dipole above a lossy earth. IEEE Trans. Antennas Propag. 2004, 52, 3133-3137.

4. Collin, R.E. Hertzian dipole radiating over a lossy earth or sea: Some early and late 20th-century controversies. Antennas Propag. Mag. IEEE 2004, 46, 64-79.

5. Sharma, A.; Singh, G. Design of single pin shorted three-dielectric-layered substrates rectangular patch microstrip antenna for communication systems. Prog. Electromagn. Res. 2008, 2, 157-165. 
6. Cella, U.M.; Johnstone, R.; Shuley, N. Electromagnetic wave wireless communication in shallow water coastal environment: Theoretical analysis and experimental results. In Proceedings of the Fourth ACM International Workshop on UnderWater Networks, Berkeley, CA, USA, 3 November 2009.

7. Wait, J.R. Electromagnetic Waves in Stratified Media: Revised Edition Including Supplemented Material; Elsevier: Amsterdam, Netherlands, 2013; Volume 3.

8. Wait, J.; Fraser, W. Radiation from a vertical dipole over a stratified ground (Part II). Trans. Ire Prof. Group Antennas Propag. 2003, 2, 144-146.

9. Wait, J.R. Radiation from a vertical antenna over a curved stratified ground. J. Res. Natl. Bur. Stand. 1956, 56, 237-244.

10. King, R.W.P.; Sandler, S.S. The electromagnetic field of a vertical electric dipole over the Earth or sea. IEEE Trans. Antennas Propag. 1994, 42, 382-389.

11. King, R.W.P. The electromagnetic field of a horizontal electric dipole in the presence of a three-layered region: Supplement. J. Appl. Phys. 1993, 74, 4845-4848.

12. Michalski, K.A.; Mosig, J.R. Multilayered media Green's functions in integral equation formulations. Antennas Propag. IEEE Trans. 2002, 45, 508-519.

13. Nikita, K.S.; Stamatakos, G.S.; Uzunoglu, N.K.; Karafotias, A. Analysis of the interaction between a layered spherical human head model and a finite-length dipole. Microw. Theory Tech. IEEE Trans. 2000, 48, 2003-2013.

14. Khamas, S.K. Electromagnetic radiation by antennas of arbitrary shape in a layered spherical media. IEEE Trans. Antennas Propag. 2009, 57, 3827-3834.

15. Quintanadíaz, G.; Menarodríguez, P.; Pérezálvarez, I.; Jiménez, E.; Dortanaranjo, B.P.; Zazo, S.; Pérez, M.; Quevedo, E.; Cardona, L.; Hernández, J. Underwater electromagnetic sensor networks-Part I: Link characterization. Sensors 2017, 17, 189.

16. Rao, S.; Wilton, D.; Glisson, A. Electromagnetic scattering by surfaces of arbitrary shape. IEEE Trans. Antennas Propag. 1982, 30, 409-418.

17. Kolundzija, B.M. Electromagnetic modeling of composite metallic and dielectric structures. IEEE Trans. Microw. Theory Tech. 1999, 47, 1021-1032.

18. Oijala, P.Y.; Taskinen, M.; Sarvas, J. Surface Integral Equation Method for General Composite Metallic and Dielectric Structures with Junctions. Prog. Electromagn. Res. 2005, 52, 81-108.

19. Cui, Z.; Han, Y.; Li, M. Solution of CFIE-JMCFIE using parallel MOM for scattering by dielectrically coated conducting bodies. J. Electromagn. Waves Appl. 2011, 25, 211-222.

20. Yang, W.; Li, K.; Li, K. A parallel solving method for block-tridiagonal equations on CPU-GPU heterogeneous computing systems. J. Supercomput. 2017, 73, 1760-1781.

21. Putnam, J.M.; Medgyesimitschang, L.N.; Gedera, M.B. Generalized method of moments for three-dimensional penetrable scatterers. Neuroimage 1994, 33, 706-714.

22. Solís, D.M.; Taboada, J.M.; Basteiro, F.O. Surface integral Equation-Method of moments with multiregion basis functions applied to plasmonics. IEEE Trans. Antennas Propag. 2015, 63, 2141-2152.

23. Rius, J.M.; Ubeda, E.; Parron, J. On the testing of the magnetic field integral equation with RWG basis functions in method of moments. Antennas Propag. IEEE Trans. 2001, 49, 1550-1553.

24. Gibson, W.C. The Method of Moments in Electromagnetics; CRC Press: Boca Raton, FL, USA, 2014.

25. Tiryaki, B. Solution of Electromagnetics Problems With the Equivalence Principle Algorithm. PhD thesis, Bilkent university, Ankara, Turkey, 2010.

26. Özgür Ergül.; Gürel, L. Improving the accuracy of the magnetic field integral equation with the linear-linear basis functions. Radio Sci. 2006, 41, 1-15.

27. Olcen, A.B. Method of Moments Analysis of an Aperture in a Thick Ground Plane. Dissertations \& Theses-Gradworks, Syracuse University, December 2012.

28. Cheng, J.; Adams, R.J.; Young, J.C.; Khayat, M.A. Augmented EFIE With Normally Constrained Magnetic Field and Static Charge Extraction. IEEE Trans. Antennas Propag. 2015, 63, 4952-4963.

(C) 2017 by the authors. Licensee MDPI, Basel, Switzerland. This article is an open access article distributed under the terms and conditions of the Creative Commons Attribution (CC BY) license (http:/ / creativecommons.org/licenses/by/4.0/). 